\title{
Association between maladaptive parenting and child self-control over time: cross-lagged study using a monozygotic twin difference design
}

\author{
Charlotte A. M. Cecil, Edward D. Barker, * Sara R. Jaffee and Essi Viding*
}

\section{Background}

Harsh parenting practices and negative parental feelings may be environmental risk factors for low self-control in children. children may also evoke certain parenting reactions.

\section{Aims}

To investigate the longitudinal relationship between parenting and self-control, as well as associated outcomes within the monozygotic (MZ) twin differences framework.

\section{Method}

Longitudinal MZ twin differences analysis was conducted on a community sample of 5184 twins using data from ages 3, 4, 7 and 9 years. Outcomes related to self-control and parenting were analysed at age 12 years.

\section{Results}

Non-shared environmental effects of parenting on the development of self-control and an evocative effect of child self-control on parenting were found. Harsh parenting predicted conduct problems for both boys and girls. Selfcontrol at age 9 predicted conduct problems and emotional difficulties at age 12 .

\section{Conclusions}

Parenting and child self-control affect one another, highlighting the potential of early interventions that target parents and children simultaneously.

\section{Declaration of interest}

None.
Lack of capacity for sustained attention, persistence and emotion regulation (i.e. self-control) has been identified as a robust predictor of psychiatric difficulties and maladaptive social and behavioural outcomes, such as increased conduct problems and anxiety. ${ }^{1-5}$ Consequently, it has been suggested that learning to control one's behaviour and emotions appropriately can be seen as a developmental milestone that has ramifications for societal well-being, and may thus constitute an important target for intervention efforts. ${ }^{6}$ Evidence suggests that maladaptive parenting, such as harsh parenting or high levels of parent-child conflict, can be a risk factor for low self-control in children. ${ }^{7}$ However, parent-child relationships are bidirectional, and it has also been documented that child characteristics may evoke maladaptive parenting. ${ }^{8}$ For example, oppositional and aggressive children can evoke parental negativity in caregivers. ${ }^{9}$ To date, few published longitudinal studies have examined bidirectional processes in selfcontrol. ${ }^{10}$ In addition, little is known about how different aspects of parenting, such as the use of disciplinary practices $v$. feelings towards the child, may relate to the development of self-control over time. There is also a need to clarify the role of parenting in self-control in early childhood, when intervention prospects may be at their peak, as self-control has been shown to develop in the first decade of life. ${ }^{7,11}$ Importantly, it is unclear whether parenting practices act as a true environmental influence, given that parents may pass on genetic risk factors for low self-control and that these same genetic risk factors could limit their own parenting competence. Thus, it is possible that the association between maladaptive parenting and lower self-control could reflect genetic rather than environmental risk. It is important to examine whether parenting affects self-control through environmental influences in order to help establish whether interventions targeting maladaptive parenting could be effective in promoting improved self-control in the child.
Our study had three main aims. First, we examined bidirectional relationships between two types of maladaptive parenting (harsh discipline and negative feelings towards the child) and self-control using the monozygotic (MZ) difference design, spanning early to middle childhood. Because MZ twins share $100 \%$ of their genes, any phenotypic differences between them will not result from genetic influences. ${ }^{12,13}$ Thus, by examining $\mathrm{MZ}$ twin differences we were able to assess the degree to which differences in maladaptive parenting affect differences in the development of self-control at ages 3, 4, 7 and 9. With regard to bidirectional effects, we also used self-control differences in MZ twins as a predictor of differences in the twins' experience of maladaptive parenting at ages 3, 4, 7 and 9. Second, we tested whether differences in self-control and parenting affect later MZ differences in conduct problems and emotional difficulties, two outcomes commonly associated with lower self-control in the child and maladaptive parenting. ${ }^{14}$ Third, we examined indirect effects between parenting, self-control and child outcomes. More specifically, we tested the degree to which parenting at age 3 years could affect conduct problems and emotional difficulties at age 12 years, through self-control. Such an indirect effect, if found, would suggest that successful intervention on maladaptive parenting could lead to lower conduct problems and emotional difficulties, through increased child self-control. We also examined the inverse: that is, whether low self-control early in development relates to increased conduct problems and emotional difficulties at age 12 years, through increased maladaptive parenting. Presence of this effect would instead suggest that a child-focused intervention could be more effective in reducing negative child outcomes by decreasing experience of maladaptive parenting. Possible gender differences for all associations were examined.

\section{Method}

\section{Participants}

We examined data from the Twins Early Development Study, an ongoing longitudinal study in which all twins born in England 
and Wales between 1994 and 1996 were identified through birth records and approached. Multiple waves of data have been collected so far ranging from 2 years to 14 years of age. The sample is reasonably representative of the general population and further details are available elsewhere. ${ }^{15}$ We assessed a subsample of 2592 MZ twin pairs (53\% girls) for whom self-control data were available from parents' ratings on at least one assessment point. Zygosity was established by means of a parent-rated instrument which has an accuracy of $95 \%$ as validated by genotyping. ${ }^{16}$

\section{Outcome measures}

\section{Self-control}

A composite measure of self-control was created using parental ratings of child characteristics at ages 3, 4, 7 and 9 years. Items were selected on the basis of measuring different dimensions of self-control, namely inattention/hyperactivity (e.g. lack of concentration, inattentive), persistence (e.g. likes to put in effort to improve, sees tasks through to the end), and emotional regulation (e.g. cries easily, tantrums). The number of items used at each collection wave varied between 8 and 11 and had high internal consistency $(\alpha=0.77-0.82)$. There was no item overlap between the composite measures of self-control and the outcome measures. An average of standardised scores was taken to create each scale at age 3, 4, 7 and 9 years. Higher scores on the self-control scales represented higher levels of self-control (a list of individual items constituting the self-control scale at each time point and information regarding the parental measures used to derive the self-control composite scale is given in an online supplement to this paper).

\section{Maladaptive parenting}

Maladaptive parenting was assessed by parental reports of harsh discipline and negative parental feelings at ages 3, 4, 7 and 9 years. Harsh discipline was measured using two questionnaire items, 'shouting' and 'smacking', adapted from a semi-structured interview. ${ }^{17}$ One parent (usually the mother) rated items on a five-point scale from 'I rarely or never do this' to 'I always do this' for their first-born twin. Items for the second-born twin were rated relative to the first-born twin using a five-point scale ranging from 'I do this a lot more' to 'I do this a lot less'. Correlations between the two items in the scale varied between 0.21 and 0.41 across time points $(P<0.001)$. Negative feelings were measured using seven items drawn from an abbreviated version of the Parental Feelings Questionnaire. ${ }^{18}$ These included statements such as 'Sometimes s/he makes me angry' and 'Sometimes I am frustrated by him/her'. Items were rated on a five-point scale ranging from 'untrue' to 'true' for the first-born twin, followed by relative ratings on a five-point scale for the second-born twin, from 'a lot more' to 'a lot less'.

Different rating measurements were used to capitalise on potential differences in parental practices between twin pairs. For both scales, ratings for the first-born twin were summed and standardised to zero mean and unit variance for the entire population. Ratings for the second-born twins were then derived from their co-twin by standardising the differential score (i.e. event occurring more or less compared with first-born twin) and adding it to the first-born twin's standardised score. Cronbach's $\alpha$ for the negative feelings scale varied between 0.59 and 0.63 across time points. For both scales higher scores represent more maladaptive parenting.

\section{Child outcomes}

Emotional and behavioural outcomes were measured at age 12 years by teacher reports of emotional difficulties and conduct problems using subscales from the Strengths and Difficulties Questionnaire. ${ }^{19}$ Each subscale was rated on a three-point scale from 'not true' to 'certainly true'. The emotional difficulties subscale comprised five items, including 'nervous or clingy in new situations, easily loses confidence' and 'many fears, easily scared'. The conduct problems scale included four items, such as 'often lies or cheats' and 'often fights with other children or bullies them'. The fifth item, 'temper tantrums', was removed from the original conduct problems scale owing to item overlap with the self-control composite measure. We examined mean standardised scores for each subscale, where higher scores indicated greater emotional and behavioural difficulties. Cronbach's $\alpha$ values were 0.77 and 0.62 for scales of emotional difficulties and conduct problems respectively.

\section{Attrition and missing data}

Inclusion in the analysis was based on there being at least one selfcontrol difference score for the twin pair. Out of the total sample of $2713 \mathrm{MZ}$ twin pairs (53\% girls), 96\% of twin pairs were included in the analysis $(n=2592)$ and $4 \%$ were excluded $(n=121)$. Using a multivariate logistic regression, we tested the extent to which the included twins differed from the excluded twins on measures of maladaptive parenting, emotional and behavioural outcomes, and found no significant difference. Thus, twin pairs included in the study did not differ significantly from twin pairs excluded from the study on any of the study measures.

\section{Statistical analysis}

We first calculated within-pair differences for the variables included in the study by subtracting each twin 2 score from the corresponding twin 1 score. In this study we controlled for nonindependence of observations (i.e. correlated scores of the twins within family) by clustering data around the family identification variable and using a maximum likelihood parameter estimate when running the statistical analysis. Missing data were handled through Full Information Maximum Likelihood. All analyses were conducted using Mplus version 6.1.1 for Windows $7 .^{20}$ For our main analysis we estimated one path analytic model using $\mathrm{MZ}$ difference scores for harsh parenting and another model using negative parental feelings. Using twin difference scores, this analytic strategy enabled us to examine the role of environmental influences in the following:

(a) the longitudinal interrelationship between parenting and self-control (cross-lagged paths, controlling for previous measurements);

(b) the unique contribution of self-control and parenting to levels of conduct problems and emotional difficulties later in childhood;

(c) the degree to which parenting can affect child outcomes indirectly, through levels of self-control (indirect effect).

Individual model fit was established using root mean square error of approximation (RMSEA; acceptable fit $\leqslant 0.08$ ), as well as the comparative fit index (CFI) and the Tucker-Lewis index ( $\mathrm{TLI}$; both indices acceptable fit $\geqslant 0.90) .{ }^{21,22}$ Indirect pathways were programmed in model constraint statements in Mplus. ${ }^{20}$ In our analyses we tested indirect effects, not mediation. Indirect effects involve the same calculations as mediation analyses, whereby the 'effect' tested is the product term of the non-standardised pathways that start from the predictor to the outcome through the intermediary variables. However, unlike mediation analyses, indirect effect analyses do not require a direct effect between two variables to be found in order to explore whether this 
association may be indirectly affected by a third intermediary variable. Because standard errors underlying indirect effects (i.e. product terms) are known to be skewed, we bootstrapped all indirect effects 10000 times with bias-corrected 95\% confidence intervals. Thus, results reported are based on the bootstrapped variability around the product of non-standardised path coefficient estimates.

It is important to note that regression (path) and correlation coefficients greater than zero indicate presence of non-shared environmental influences. Non-significant coefficients do not necessarily represent a lack of effect at a phenotypic level, but rather demonstrate that the association in question is not environmentally mediated to a significant extent. Furthermore, because all analyses were conducted using MZ differences scores, results are discussed in terms of the sign of the path coefficients (i.e. negative or positive), so that, for example, a negative coefficient implies that increasingly positive MZ differences values in one variable predict increasingly negative $M Z$ differences in the other variable. We also examined possible gender differences for individual path coefficients by calculating how well the model fitted the data when parameters for a given path coefficient were free to vary across gender $v$. fixed to remain equal across gender. Gender differences in the indirect pathways were tested by bootstrapping the difference in the respective pathways within a multiple group model (i.e. gender as the group).

\section{Results}

Two cross-lagged models were estimated, one using MZ difference scores in harsh parenting and self-control and another using MZ difference scores in negative parental feelings and selfcontrol. Both models fit the data adequately: harsh parenting: $\chi^{2}(24)=85.81, P<0.001$, CFI $=0.97$, TLI $=0.94$, RMSEA $=0.02$ (90\% CI 0.017-0.027); negative parental feelings: $\chi^{2}(24)=$ 97.78, $P<0.001, \quad$ CFI $=0.97, \quad$ TLI $=0.94, \quad$ RMSEA $=0.02 \quad(90 \%$ CI $0.019-0.029$ ).

\section{Step 1: Self-control and maladaptive parenting}

Harsh parenting

Results revealed a significant bidirectional effect of differences in self-control and harsh parenting in early childhood (Fig. 1). More specifically, twins who experienced higher levels of harsh parenting at age 3 years had lower levels of self-control at age 4 years than their co-twin, above and beyond baseline differences in self-control. Similarly, twins who had higher levels of self-control at age 3 years experienced lower levels of harsh parenting at age 4 years compared with their co-twin, above and beyond baseline differences in the experience of harsh parenting. Thus, during early childhood, experience of higher levels of harsh parental discipline result in lower self-control during subsequent development and, in turn, lower self-control in children can evoke more harsh responses of parental discipline. This bidirectional effect continued into mid-childhood (Fig. 1). Between the ages of 7 and 9 years, the twin with higher self-control received significantly less harsh parental responses; however, harsh parenting differences at age 7 years no longer predicted differences in self-control at age 9 years, so that the twin receiving harsher parenting at 7 years of age was not significantly lower in selfcontrol at age 9 years. Comparing the magnitude of effects between male and female twin pairs for each significant path coefficient did not reveal any significant gender difference; thus, the longitudinal association between self-control differences and differences in harsh parenting was found to be similar across boys and girls.

\section{Negative parental feelings}

The relationship between differences in the experience of negative parental feelings and self-control differences is shown in Fig. 2. The twin who experienced more negative feelings at age 3 years had lower levels of self-control at age 4 years above and beyond baseline differences in self-control. Similarly, the twin who experienced more negative parental feelings at age 4 years had lower self-control at age 7 years. This effect was no longer significant between ages 7 and 9 years. With regard to child evocative processes, self-control differences at age 3 years did not predict differences in the experience of negative parental feelings at age 4 years. However, self-control differences at age 4 years did predict differences in negative parental feelings at age 7 years. That is, parents reported more negative feelings at age 7 years about the twin who had lower self-control at age 4 years. This association was no longer significant between the ages of 7 and 9 years. Thus, there appears to be a significant bidirectional relationship between differences in negative parental feelings and self-control differences only between the ages of 4 and 7 years.

Age 3

Age 4

Age 7

Age 9

Age 12

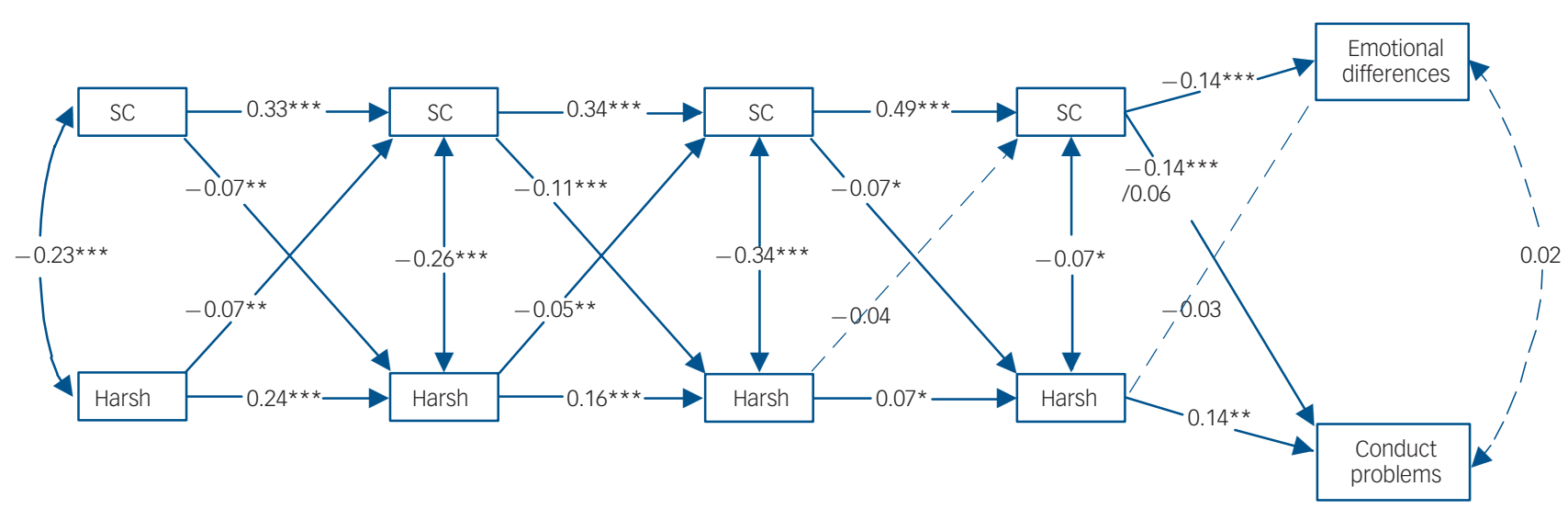

Fig. 1 Relationship between differential experience of harsh parenting and self-control differences from early to middle childhood.

Double-arrow lines indicate standardised correlation coefficients. Single-arrow lines indicate standardised path coefficients. Dotted arrow lines indicate non-significant coefficients. Paths with two coefficients indicate presence of a significant gender difference (male/female coefficient). $n=5184$. Harsh, harsh parenting; SC, self-control. Results are significant at ${ }^{* *} P<0.001,{ }^{* *} P<0.01,{ }^{*} P<0.05$ 


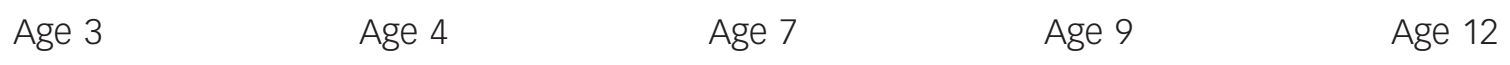

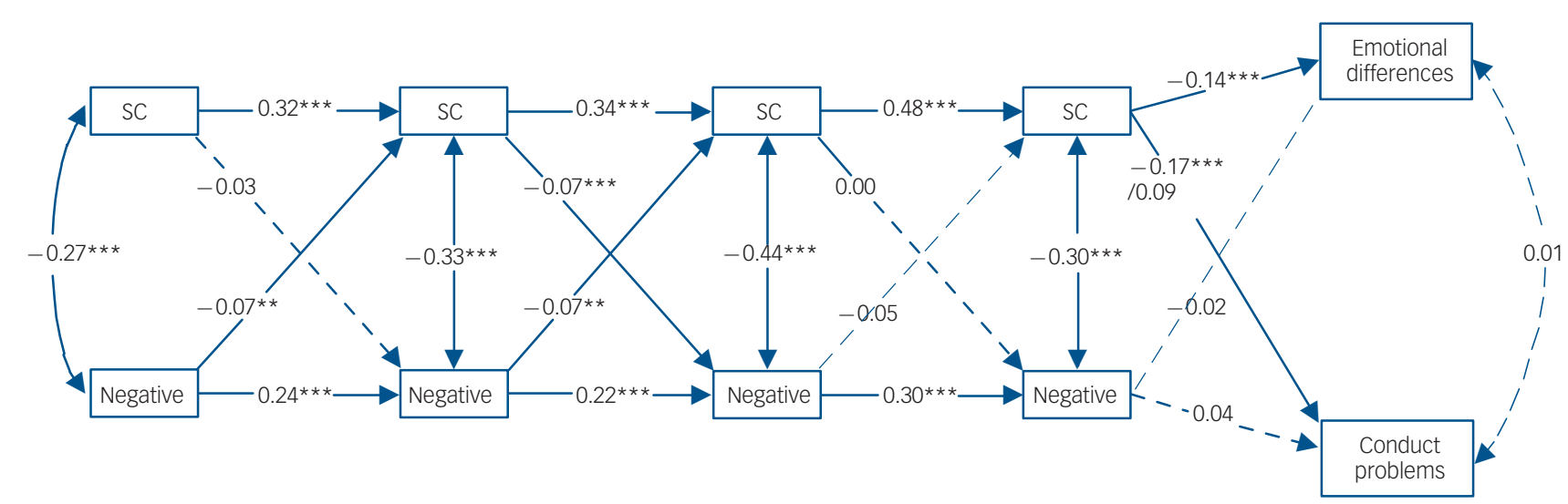

Fig. 2 Relationship between differential experience of negative parental feelings and self-control differences from early to mid childhood.

Double-arrow lines indicate standardised correlation coefficients. Single-arrow lines indicate standardised path coefficients. Dotted arrow lines indicate non-significant coefficients. Paths with two coefficients indicate presence of a significant gender difference (male/female coefficient). $n=5180$. Negative, negative parental feelings; SC, self-control. Results are significant at ${ }^{* \star *} P<0.001,{ }^{* \star P} P<0.01,{ }^{*} P<0.05$.

Comparing the magnitude of effects between male and female twin pairs for each significant path coefficient revealed no significant gender difference in the longitudinal association between negative parental feelings and child self-control differences.

\section{Step 2: Child outcomes at age 12 years}

\section{conduct problems}

The twins who experienced more harsh discipline at 9 years of age had significantly more conduct problems at age 12 years compared with their co-twins (Fig. 1). There was no gender difference for this association. Differences in the experience of negative parental feelings at age 9 years did not predict within-pair differences in conduct problems at age 12 years. In both parenting models, the twins with higher levels of self-control at age 9 years had significantly lower levels of conduct problems at age 12 years compared with their co-twins. When we examined gender differences for these associations, the association between selfcontrol at age 9 years and conduct problems at age 12 years was statistically significant for boys but not for girls (within harsh parenting model $\Delta \chi^{2}(1)=7.07, P<0.01$; within negative parental feelings model $\left.\Delta \chi^{2}(1)=13.62, P<0.001\right)$.

\section{Emotional difficulties}

Neither differences in harsh parenting nor negative parental feelings at age 9 years were associated with differences in emotional difficulties at age 12 years. Across both parenting models twins who had higher levels of self-control at age 9 years also had significantly lower levels of emotional difficulties at age 12 years than their co-twins. There was no gender difference for this association.

\section{Step 3: Indirect effects between parenting, self-control and child outcomes}

We tested the extent to which parenting might affect child outcomes indirectly, through levels of self-control. In turn, we also examined the degree to which self-control (at age 3) might indirectly affect child outcomes (at age 12), through parenting (at ages 4, 7 and 9 years). Two significant indirect effects were identified using bootstrapped non-standardised estimates, and these related exclusively to the model of negative parental feelings (see online Figs DS1 and DS2 for models showing the non-standardised estimates). First, greater experience of negative parental feelings at 3 years of age significantly increased conduct problems at age 12 years, through self-control differences at ages 4,7 and 9 years $(b=0.004$, s.e. $=0.002, P=0.039)$. This indirect pathway was significant for male twin pairs only (difference between boys and girls: $b=0.005$, s.e. $=0.002, P=0.002$ ). Second, more negative parental feelings at age 3 years significantly increased emotional difficulties at age 12 years, through selfcontrol differences at ages 4,7 and 9 years $(b=0.004$, s.e. $=0.002, P=0.039)$. This indirect pathway did not significantly differ according to gender. Harsh parenting was not found to affect child outcomes indirectly through intermediate levels of self-control. At the same time, self-control differences at age 3 years did not indirectly predict differences in outcomes at age 12 years through levels of harsh parenting or negative parental feelings at ages 4, 7 and 9 years.

\section{Discussion}

This study was the first, to our knowledge, to explore bidirectional associations between parenting and self-control from early to middle childhood making use of the MZ twin difference design. Differences in self-control were found to be stable over time and driven to a significant extent by differential experience of harsh parenting and negative parental feelings in early childhood. Early parenting was, therefore, found to be an important non-shared environmental influence in the development of self-control differences in children. At the same time, differences in selfcontrol were also found to predict differential experience of maladaptive parenting, thus supporting the idea of a bidirectional relationship between the child and the parent.

We also examined the relationship between differences in maladaptive parenting and self-control at age 9 years and child outcomes at age 12 years. The twin who received harsher parenting at age 9 years was at increased risk of conduct problems but not emotional difficulties at age 12 years. Differences in the experience of negative parental feelings at age 9 years did not predict differences in either child outcome. The twin with lower self-control at age 9 years had greater emotional difficulties at age 12 years among both boys and girls. For male twin pairs only, 
lower self-control at age 9 years predicted greater conduct problems at age 12 years. Finally, we demonstrated that differential experiences of negative parental feelings at age 3 years increased the risk of boys' conduct problems and boys' and girls' emotional difficulties at age 12 years through a pathway involving low selfcontrol at 4, 7 and 9 years. That is, once controlling for age 3 years, negative parental feelings at age 9 years did not directly predict conduct problems and emotional difficulties; rather, negative parental feelings indirectly predicted conduct problems (boys only) and emotional difficulties through low self-control. Such a finding indicates the importance of the emotional quality of the early caregiving environment for the development of self-control in children.

\section{Parenting as a non-shared environmental influence on self-control}

The use of the MZ twin differences design provided a valuable opportunity to elucidate further the role of differential parenting in the development of self-control differences in genetically identical individuals. Our findings document a longitudinal, non-shared environmental effect of early parenting, whereby the twins who experienced harsher parental discipline and more negative parental feelings also were lower in self-control. These findings clarify phenotypic-level results from previous studies that show parenting to be an important predictor of self-control differences, and demonstrate that this effect is, at least partially, environmentally mediated. ${ }^{7}$ It is noteworthy that a significant effect of differential parenting on subsequent self-control differences was observed until age 7 years, but not between ages 7 and 9 years. This suggests that the effects of differential parenting on self-control development are particularly relevant during early childhood.

Evidence that discordant parenting in early childhood can cause stable differences over time in twins' level of self-control underscores the potential of parenting programmes in driving positive effects on child levels of self-control. Our study extends previous research findings by identifying specific parental practices that are salient to the development of self-control and that represent a child-specific environmental risk. Importantly, the study distinguishes between a direct/behavioural (i.e. harsh parenting) and an emotional/parental perception (i.e. negative parental feelings) component of parenting. Other types of parenting may also be important predictors of self-control differences. For example, experiencing inconsistent routines (bed, meal times) and inconsistent punishment can increase the likelihood of early self-control difficulties (unpublished data, details available from the author on request). Further research is needed to identify additional parenting practices that are nonshared environmental risk factors for the development of maladaptive self-control.

\section{Child self-control and evoked parental response}

Our study was also the first to document a longitudinal evocative process, whereby differences in child self-control were found to predict differences in reported use of both harsh parental disciplinary behaviours and negative parental feelings. The magnitude of this effect differed across developmental stages and type of maladaptive parenting, but was particularly consistent with regard to differences in self-control evoking differences in harsh parental disciplinary behaviours. These results are consistent with previous developmental studies suggesting that temperament and effortful control may evoke certain parental reactions. ${ }^{23}$ The results reported here may also suggest that low self-control in children can exacerbate maladaptive parenting responses, so that child-centred strategies could be used to scaffold positive parenting interventions. It is possible that differences in selfcontrol that predict differences in parenting can themselves be driven by earlier instances of maladaptive parenting or adverse environmental conditions. Future studies should assess the role of perturbations in the prenatal and early postnatal environments that may affect the early development of self-control in the child.

\section{Effect of parenting and self-control on child outcomes}

Our findings demonstrated that harsh parenting at age 9 years increased the risk of conduct problems but not emotional difficulties at age 12 years. Negative parental feelings at age 9 years did not predict later emotional difficulties and conduct problems. Self-control differences predicted differences in both conduct problems and emotional difficulties at age 12 years. In the case of conduct problems this association was specific for boys, and may suggest a child-driven process by which boys may be more vulnerable to developing conduct problems than girls. Together, these results are consistent with previous studies identifying both self-control and harsh parenting as important markers of later psychological well-being and individual success. ${ }^{3,4}$ Future research is needed to explore the mechanisms by which levels of selfcontrol and types of parenting predict outcomes in a number of areas of child functioning.

We additionally tested the degree to which maladaptive parenting in early childhood might increase later conduct problems and emotional difficulties, through intermediate measures of low self-control; and conversely, the extent to which self-control at age 3 years could lead to greater conduct problems and emotional difficulties through experience of maladaptive parenting at ages 4, 7 and 9 years. Greater experience of harsh parenting at age 3 years did not indirectly increase negative child outcomes at age 12 years through intermediate levels of child selfcontrol. On the other hand, greater experience of negative parental feelings at age 3 years increased both risk of conduct problems and emotional difficulties at age 12 years through decreased levels of self-control from ages 4 to 9 years. With regard to conduct problems, this indirect effect was significant for male twin pairs only. Again, this suggests a possible pathway by which boys may be more vulnerable to develop conduct problems than girls, as these may result from multiple risk factors, including low levels of child self-control and experience of harsh parenting at age 9 years, but also indirectly through experience of negative parental feelings at age 3 years. Thus, it seems that differences in negative parental feelings act as catalysts towards differential self-control development, which in turn increases risk of greater conduct problems for boys and greater emotional difficulties in both genders.

Consistent with previous findings, the results suggest that harsh parenting exerts an effect on child conduct problems by a more 'direct' and immediate route. ${ }^{24}$ These results extend previous findings by showing that although negative parental feelings do not appear to have a direct effect on conduct problems, they do act indirectly to increase both conduct problems and emotional difficulties through levels of self-control. Thus, parent-centred interventions targeting negative parental feelings during the child's early development could lead to lower risk of later childhood psychopathology, through increased levels of child self-control. It is not possible to tell from the data presented here why harsh parenting and negative parental feelings have their effect on conduct problems through 'direct' and 'indirect' routes respectively. The 'direct' route is likely to reflect a number of processes, such as modelling aggressive behaviour to the child or making the child feel threatened and thus increasing the likelihood 
of defensive aggressive reactions in the child. ${ }^{25,26}$ Any account of the 'indirect' route is even more speculative, but it is possible to postulate that the child who experiences more negative parental feelings is under more emotional stress, which might in turn have an impact on the cognitive and emotional resources that the child has available for developing effortful regulation strategies.

\section{Considerations for intervention}

Findings from our study highlight early childhood as a promising window of opportunity for enhancing levels of self-control, and support the notion that interventions that 'start at the beginning' are likely to produce greater benefits than the implementation of remedial interventions later on. ${ }^{27}$ Baseline measures of self-control in this study were recorded at age 3 years, an important period for the formation of social and emotional competencies, which relies on the ability of a parent to meet the child's needs in order for the child to achieve these developmental milestones. ${ }^{28}$ Evidence from studies looking at development in utero and during the first 2 years of life demonstrate that prenatal and early postnatal adversity increases the risk of negative outcomes in the child. ${ }^{29,30}$ More research is needed to establish which other factors in the child's environment prior to the age of 3 years can affect the development of self-control. Evidence that parenting and child self-control can affect one another, especially early in childhood, not only highlights the potential of interventions that 'start at the beginning' but also emphasises the need for programmes that target both parents and children concurrently. ${ }^{31}$

\section{Limitations}

The study has a number of limitations. First, both ratings of maladaptive parenting and self-control were recorded using parental reports, which could raise the possibility of shared method variance. Future studies examining the relationship between parenting and self-control should aim to use multiple informants, to provide additional information on differences within $\mathrm{MZ}$ twin pairs. Second, the early environment was measured using ratings of negative parental feelings and harsh parental discipline, which comprised only two items. It would be important to replicate the present results using a more comprehensive assessment of harsh disciplinary parental behaviour. Moreover, a measure of positive parenting could have been included as an additional environmental influence on the development of selfcontrol, enabling us to observe the effect of positive parenting on children with lower baseline levels of self-control. Other non-shared environmental risk factors besides parenting could have also been explored, and future research should consider different risk factors that are salient at different developmental eras. The inclusion of a wider range of interrelated risk factors might also help strengthen model fit, which in our study was only adequate. Importantly, there is a need to understand why parents of an identical twin pair would treat one twin differently from the other. Some of these differences may be explained by additional sources of phenotypic variation, such as differences in gene expression resulting from child-specific epigenetic mechanisms. ${ }^{32}$ Future studies should attempt to clarify the role of these influences on parenting. Third, it is possible that our findings may not generalise to dizygotic twin pairs or singletons. It would be useful to replicate the study using different populations of twins and non-twins. The present cross-lagged paths were relatively weak and it is possible that effect sizes might be found to be larger for singletons in future epidemiological and longitudinal studies. With regard to child outcomes, the study included teacher-rated outcomes related to emotional and behavioural difficulties. Future research should aim to use multiple sources of information (e.g. clinical assessments) at multiple time points to obtain more robust outcome measures. Finally, the addition of other intermediary outcomes, such as school readiness and early engagement in risk-taking behaviour, might also contribute to a more comprehensive assessment of the developmental pathways of low self-control children. ${ }^{14}$

\section{Charlotte A. M. Cecil, MSC, Division of Psychology and Language Sciences, University College London; Edward D. Barker, PhD, Department of Psychological Sciences, Birkbeck College, University of London; Sara R. Jaffee, PhD, Medical Research Council Social, Genetic and Developmental Psychiatry Centre, Institute of Psychiatry, London; Essi Viding, PhD, Division of Psychology and Language Sciences, University College London, UK \\ Correspondence: Essi Viding, Division of Psychology and Language Sciences, University College London, 26 Bedford Way, London WC1H OAP, UK. Email: e.viding@ucl.ac.uk \\ First received 13 Dec 2011, final revision 22 May 2012, accepted 29 May 2012}

\section{Funding}

The Twins Early Development Study receives support from the UK Medical Research Council (G0500079). C.A.M.C. was supported by Kids-Company charity studentship during the writing of the article.

\section{Acknowledgements}

The authors are indebted to the twins, their parents and teachers, and acknowledge Professor Robert Plomin for his generous support of this work.

\section{References}

1 Heckman JJ. Skill formation and the economics of investing in disadvantaged children. Science 2006; 312: 1900-2.

2 Miller HV, Barnes JC, Beaver KM. Self-control and health outcomes in a nationally representative sample. Am J Health Behav 2011; 35: 15-27.

3 Moffitt TE, Arseneault L, Belsky D, Dickson N, Hancox RJ, Harrington HL, et al. A gradient of childhood self-control predicts health, wealth, and public safety. Proc Natl Acad Sci USA 2011; 108: 2693-8.

4 Tangney JP, Baumeister RF, Boone AL. High self-control predicts good adjustment, less pathology, better grades, and interpersonal success. J Pers 2004; 72: 271-324.

5 Valiente C, Lemery-Chalfant K, Swanson J, Reiser M. Prediction of children's academic competence from their effortful control, relationships, and classroom participation. J Educ Psychol 2008; 100: 67-77.

6 Shonkoff JPE, Phillips DA. From Neurons to Neighborhoods: The Science of Early Child Development. National Academy Press, 2000.

7 Vazsonyi AT, Huang L. Where self-control comes from: on the development of self-control and its relationship to deviance over time. Dev Psychol 2010; 46: $245-57$.

8 Burt SA, Krueger RF, McGue M, lacono W. Parent-child conflict and the comorbidity among childhood externalizing disorders. Arch Gen Psychiatry 2003; 60: 505-13.

9 Larsson H, Viding E, Rijsdijk FV. Relationships between parental negativity and childhood antisocial behavior over time: a bidirectional effects model in a longitudinal genetically informative design. J Abnorm Child Psychol 2008; 36: $633-45$

10 Eisenberg N, Spinrad TL, Eggum ND. Emotion-related self-regulation and its relation to children's maladjustment. Annu Rev Clin Psychol 2010; 6: 495-525.

11 Piquero AR, Jennings WG, Farrington DP. On the malleability of self-control: theoretical and policy implications regarding a general theory of crime. Justice Q 2010; 27: 803-34.

12 Plomin R, Daniels $D$. Why are children in the same family so different from one another? Int J Epidemiol 2011; 40: 563-82.

13 Vitaro F, Brendgen M, Arseneault L. The discordant MZ-twin method: one step closer to the holy grail of causality. Int J Behav Dev 2009; 33: 376-82.

14 Eisenberg N, Valiente C, Eggum ND. Self-regulation and school readiness. Early Educ Dev 2010; 21: 681-98.

15 Trouton A, Spinath FM, Plomin R. Twins Early Development Study (TEDS): a multivariate, longitudinal genetic investigation of language, cognition and behavior problems in childhood. Twin Res 2002; 5: 444-8. 
16 Price TS, Freeman B, Craig I, Petrill SA, Ebersole L, Plomin R. Infant zygosity can be assigned by parental report questionnaire data. Twin Res 2000; 3: 129-33.

17 Deater-Deckard K, Dodge KA, Bates JE, Pettit GS. Multiple risk factors in the development of externalizing behavior problems: group and individual differences. Dev Psychopathol 1998; 10: 469-93.

18 Deater-Deckard K, Pike A, Petrill SA, Cutting AL, Hughes C, O'Connor TG. Nonshared environmental processes in social-emotional development: an observational study of identical twin differences in the preschool period. Dev Sci 2001; 4: F1-6.

19 Goodman R. Psychometric properties of the strengths and difficulties questionnaire. J Am Acad Child Adolesc Psychiatry 2001; 40: 1337-45.

20 Muthén LK, Muthén BO. Mplus User's Guide. Muthén \& Muthén, 1998.

21 Bentler PM, Bonett, DG. Significance tests and goodness of fit in the analysis of covariance structures. Psychol Bull 1980; 88: 588-606.

22 Browne MW, Cudeck R. Alternative ways of assessing model fit. Sociol Method Res 1992; 21: 230-58.

23 Lengua L. Growth in temperament and parenting as predictors of adjustment during children's transition to adolescence. Dev Psychol 2006; 42: 819-32.

24 Barker ED, Maughan B. Differentiating early-onset persistent versus childhood-limited conduct problem youth. Am J Psychiatry 2009; 166: 900-8.
25 Bandura A. Social learning theory of aggression. In Control of Aggression: Implications from Basic Research (ed. JG Knutson): 201-50. Aldine-Atherton, 1971.

26 Frick PJ, Viding E. Antisocial behavior from a developmental psychopathology perspective. Dev Psychopathol 2009; 21: 1111-31.

27 Tremblay RE. Prevention of youth violence: why not start at the beginning? J Abnorm Child Psychol 2006; 34: 481-7.

28 Jaffee SR. Sensitive, stimulating caregiving predicts cognitive and behavioral resilience in neurodevelopmentally at-risk infants. Dev Psychopathol 2007; 19: 631-47.

29 Barker ED, Jaffee SR, Uher R, Maughan B. The contribution of prenatal and postnatal maternal anxiety and depression to child maladjustment. Depress Anxiety 2011; 28: 696-702.

30 Gluckman PD, Cutfield W, Hofman P, Hanson MA. The fetal, neonatal, and infant environments - the long-term consequences for disease risk. Early Hum Dev 2005; 81: 51-9.

31 Lochman JE, Wells KC. The coping power program at the middle-school transition: universal and indicated prevention effects. Psychol Addict Behav 2002; 16: S40-54.

32 Fraga MF, Ballestar E, Paz MF, Ropero S, Setien F, Ballestar ML, et al. Epigenetic differences arise during the lifetime of monozygotic twins. Proc Natl Acad Sci USA 2005; 102: 10604-9.
Mindfulness

Anthony Bateman

The term mindfulness signals a focus on mind rather than behaviour. As explicated in Bhuddism, mindfulness is enhanced attention to, and emotionally detached awareness of, current experience, requiring openness to sensation without judgement. It is not restricted to any object or function. One can be mindful of a flower, of one's breathing or of another's behaviour. Practising mindfulness improves depression and emotional regulation through detachment, changing not thoughts and feelings but the person's relationship to them. We all need to be mindful in our everyday work. The psychiatrist, of both his and his patient's mind; but then he is mentalising. 